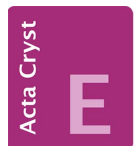

\section{Crystal structure of 5-diethylamino-2- (\{[4-(diethylamino)phenyl]imino\}methyl)- phenol}

\author{
C. Vidya Rani, ${ }^{\text {a }}$ G. Chakkaravarthi, ${ }^{\text {b* N. Indra Gandhi }}{ }^{\mathrm{c} *}$ \\ and G. Rajagopal ${ }^{\mathrm{a}}$
}

a PG \& Research Department of Chemistry, Chikkanna Government Arts College, Tiruppur 641 602, India, 'bepartment of Physics, CPCL Polytechnic College,

Chennai 600 068, India, and ' PG \& Research Department of Chemistry, Presidency College (Autonomous), Chennai 600 005, India. *Correspondence e-mail:

chakkaravarthi_2005@yahoo.com, jothivenkateswaran@yahoo.co.in

Received 1 September 2015; accepted 3 September 2015

Edited by V. Rybakov, Moscow State University, Russia

In the title compound, $\mathrm{C}_{21} \mathrm{H}_{29} \mathrm{~N}_{3} \mathrm{O}$, the dihedral angle between the planes of the aromatic rings is $8.1(2)^{\circ}$. The ethyl groups at one terminal site of the compound are disordered over two sets of sites with occupancies of 0.775 (9) and 0.225 (9). The molecule has an $E$ conformation about the $\mathrm{N}=\mathrm{C}$ bond. The molecular structure features an intramolecular $\mathrm{O}-\mathrm{H} \cdots \mathrm{N}$ hydrogen bond, which closes an $S(6)$ loop. In the crystal, weak $\mathrm{C}-\mathrm{H} \cdots \pi$ interactions leads to the formation of a threedimensional network.

Keywords: crystal structure; phenol; Schiff base; intramolecular hydrogen bond; $\mathrm{C}-\mathrm{H} \cdots \pi$ interactions; biological activity; pharmacological activity.

CCDC reference: 1422036

\section{Related literature}

For biological and pharmacological activities of Schiff base compounds and their derivatives, see: Khandar et al. (2005); Chen et al. (2006); Kidwai et al. (2000). For similar structures, see: Manvizhi et al. (2011); Thirugnanasundar et al. (2011); Rani et al. (2015).

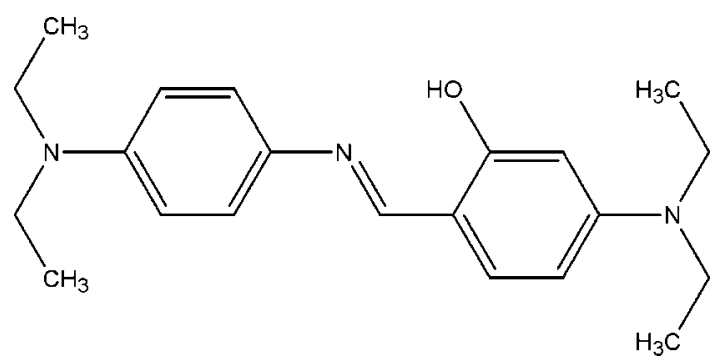

\section{Experimental}

\subsection{Crystal data}

$\mathrm{C}_{21} \mathrm{H}_{29} \mathrm{~N}_{3} \mathrm{O}$

Orthorhombic, $P 2_{1} 2_{1} 2_{1}$

$a=8.1986$ (4) §

$b=9.7128(4) \AA$

$c=24.4172(12) \AA$

$$
\begin{aligned}
& V=1944.38(16) \AA^{3} \\
& Z=4 \\
& \text { Mo } K \alpha \text { radiation } \\
& \mu=0.07 \mathrm{~mm}^{-1} \\
& T=295 \mathrm{~K} \\
& 0.28 \times 0.26 \times 0.24 \mathrm{~mm}
\end{aligned}
$$

\subsection{Data collection}

Bruker Kappa APEX II CCD diffractometer

Absorption correction: multi-scan (SADABS; Sheldrick, 1996)

$T_{\min }=0.980, T_{\max }=0.983$

29557 measured reflections 3556 independent reflections 2130 reflections with $I>2 \sigma(I)$ $R_{\text {int }}=0.046$

\subsection{Refinement}

$R\left[F^{2}>2 \sigma\left(F^{2}\right)\right]=0.064$

$w R\left(F^{2}\right)=0.205$

$S=1.07$

3556 reflections

272 parameters

10 restraints
$\mathrm{H}$ atoms treated by a mixture of independent and constrained refinement
$\Delta \rho_{\max }=0.44 \mathrm{e} \AA^{-3}$
$\Delta \rho_{\min }=-0.20$ e $\AA^{-3}$

Table 1

Hydrogen-bond geometry $\left(\AA{ }^{\circ}\right)$.

$C g 1$ and $C g 2$ are the centroids of the $\mathrm{C} 5-\mathrm{C} 10$ and $\mathrm{C} 12-\mathrm{C} 17$ rings, respectively.

\begin{tabular}{lllll}
\hline$D-\mathrm{H} \cdots A$ & $D-\mathrm{H}$ & $\mathrm{H} \cdots A$ & $D \cdots A$ & $D-\mathrm{H} \cdots A$ \\
\hline $\mathrm{O} 1-\mathrm{H} 1 \cdots \mathrm{N} 2$ & $0.86(2)$ & $1.81(4)$ & $2.563(5)$ & $144(6)$ \\
$\mathrm{C} 18-\mathrm{H} 18 A \cdots C g 2^{\mathrm{i}}$ & 0.97 & 2.92 & $3.660(5)$ & 134 \\
$\mathrm{C} 1 A-\mathrm{H} 1 A 1 \cdots C g 1^{\mathrm{ii}}$ & 0.96 & 2.80 & $3.49(4)$ & 130
\end{tabular}

Symmetry codes: (i) $-x+\frac{5}{2},-y-1, z+\frac{1}{2}$; (ii) $-x-1, y+\frac{3}{2},-z+\frac{1}{2}$.

Data collection: APEX2 (Bruker, 2004); cell refinement: SAINT (Bruker, 2004); data reduction: $S A I N T$; program(s) used to solve structure: SHELXS97 (Sheldrick, 2008); program(s) used to refine structure: SHELXL97 (Sheldrick, 2008); molecular graphics: PLATON (Spek, 2009); software used to prepare material for publication: SHELXL97 and PLATON (Spek, 2009).

\section{Acknowledgements}

The authors acknowledge the SAIF, IIT, Madras, for the data collection. 
Supporting information for this paper is available from the IUCr electronic archives (Reference: RK2432).

\section{References}

Bruker (2004). APEX2 and SAINT. Bruker AXS Inc., Madison, Wisconsin, USA.

Chen, Y., Zhao, Y., Lu, C., Tzeng, C. \& Wang, J. (2006). Bioorg. Med. Chem. 14, 4373-4378.
Khandar, A. A., Hosseini-Yazdi, S. A. \& Zarei, S. A. (2005). Inorg. Chim. Acta, 358, 3211-3217.

Kidwai, M., Bhushan, K., Sapra, P., Saxena, R. \& Gupta, R. (2000). Bioorg. Med. Chem. 8, 69-72.

Manvizhi, K., Chakkaravarthi, G., Anbalagan, G. \& Rajagopal, G. (2011). Acta Cryst. E67, o2500.

Rani, C. V., Chakkaravarthi, G. \& Rajagopal, G. (2015). Acta Cryst. E71, o503. Sheldrick, G. M. (1996). SADABS. University of Göttingen, Germany.

Sheldrick, G. M. (2008). Acta Cryst. A64, 112-122.

Spek, A. L. (2009). Acta Cryst. D65, 148-155.

Thirugnanasundar, A., Suresh, J., Ramu, A. \& RajaGopal, G. (2011). Acta Cryst. E67, o2303. 


\section{supporting information}

Acta Cryst. (2015). E71, o712-o713 [doi:10.1107/S205698901501645X]

\section{Crystal structure of 5-diethylamino-2-(\{[4-(diethylamino)phenyl]imino\}methyl)- phenol}

\section{Vidya Rani, G. Chakkaravarthi, N. Indra Gandhi and G. Rajagopal}

\section{S1. Comment}

Schiff base derivatives serve as intermediates in certain enzymatic reactions and are also found in proteins that form the connective tissue (Khandar et al., 2005; Chen et al., 2006) and in the pharmaceutical field (Kidwai et al., 2000). We herein report the crystal structure of the title compound (Fig.1). The geometric parameters of the title compound are comparable to the reported structures (Manvizhi et al., 2011; Thirugnanasundar et al., 2011; Rani et al., 2015). The dihedral angle between the rings (C5-C10) and (C12-C17) is $8.1(2)^{\circ}$. The ethyl groups at one terminal site (N1) of the compound are disordered over two positions, with the site occupancies of 0.775 (9) and 0.225 (9). The molecular structure is stabilized by weak intramolecular $\mathrm{O}-\mathrm{H} \cdots \mathrm{N}$ hydrogen bond (Table 1). The crystal structure is influenced by weak $\mathrm{C}-\mathrm{H}^{\cdots} \pi$ (Table 1) interactions to form a three dimensional network.

\section{S2. Experimental}

For the preparation of Schiff base, an ethanolic solution (10 ml) of 5-(diethylamino)-2-hydroxybenzaldehyde (0.5 mol) and the same volume of ethanolic solution of $N, N$-diethylbenzene-1,4-diamine $(0.5 \mathrm{~mol})$ are mixed. The solution is mixed on magnetic stirrer with addition of 2 to 3 drops of glacial acetic acid. The reaction mixture is refluxed for $2 \mathrm{hrs}$ and allowed to cool down to room temperature, crystalline solid precipitate from the mixture is separated out. Crystalline products are washed with ice cold ethanol and dried in vacuo over anhydrous $\mathrm{CaCl}_{2}$. Single crystals suitable for the $\mathrm{X}$-ray diffraction are obtained by slow evaporation of a solution of the title compound in $D M F$ at room temperature.

\section{S3. Refinement}

The $\mathrm{H}$ atoms were positioned geometrically and refined using riding model with $\mathrm{C}-\mathrm{H}=0.93 \AA$ and $U_{\text {iso }}(\mathrm{H})=1.2 U_{\text {eq }}(\mathrm{C})$ for aromatic $\mathrm{H}, \mathrm{C}-\mathrm{H}=0.97 \AA$ and $U_{\text {iso }}(\mathrm{H})=1.2 U_{\text {eq }}(\mathrm{C})$ for $\mathrm{CH}_{2}, \mathrm{C}-\mathrm{H}=0.96 \AA$ and $U_{\text {iso }}(\mathrm{H})=1.5 U_{\text {eq }}(\mathrm{C})$ for $\mathrm{CH}_{3} . \mathrm{H}$ atom for $\mathrm{O}$ atom is found from Fourier map and refined freely with $U_{\text {iso }}(\mathrm{H})=1.5 U_{\mathrm{eq}}(\mathrm{O})$ and distance restraint $0.82 \AA$. The components of the anisotropic displacement parameters in the direction of the bond between $\mathrm{C} 9$ and $\mathrm{O} 1$ were restrained to be equal within an effective standard deviation of 0.001 using the DELU command. The $\mathrm{N} 1-\mathrm{C} 2, \mathrm{~N} 1-\mathrm{C} 3, \mathrm{~N} 1-\mathrm{C} 2 \mathrm{~A}$, $\mathrm{N} 1-\mathrm{C} 3 \mathrm{~A}$ distances were restraint to $1.46(1) \AA$ and $\mathrm{C} 1-\mathrm{C} 2, \mathrm{C} 1 \mathrm{~A}-\mathrm{C} 2 \mathrm{~A}, \mathrm{C} 3-\mathrm{C} 4$ and $\mathrm{C} 3 \mathrm{~A}-\mathrm{C} 4 \mathrm{~A}$ distances were restraint to $1.53(1) \AA$ 


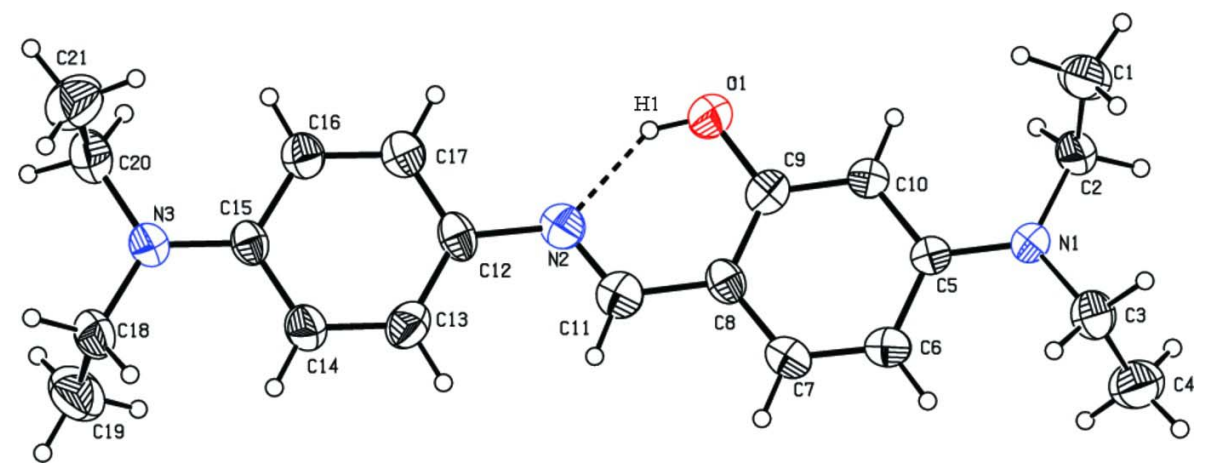

\section{Figure 1}

The molecular structure of title compaund, with the atom-numbering scheme. Displacement ellipsoids are drawn at the $30 \%$ probability level. $\mathrm{H}$ atoms are presented as a small spheres of arbitrary radius. The intramolecular hydrogen bond is depicted by a dashed line. Only the major occupancy component of the disordered diethylamino-group $\left[-\mathrm{N} 1\left(\mathrm{C}_{2} \mathrm{H}_{5}\right)_{2}\right]$ is shown.

\section{5-Diethylamino-2-(\{[4-(diethylamino)phenyl]imino\}methyl)phenol}

\section{Crystal data}

$\mathrm{C}_{21} \mathrm{H}_{29} \mathrm{~N}_{3} \mathrm{O}$

$M_{r}=339.47$

Orthorhombic, $P 22_{1} 2_{1} 2_{1}$

Hall symbol: P 2ac 2ab

$a=8.1986$ (4) $\AA$

$b=9.7128(4) \AA$

$c=24.4172(12) \AA$

$V=1944.38(16) \AA^{3}$

$Z=4$

\section{Data collection}

Bruker Kappa APEX II CCD diffractometer

Radiation source: fine-focus sealed tube Graphite monochromator $\omega$ and $\varphi$ scans

Absorption correction: multi-scan

(SADABS; Sheldrick, 1996)

$T_{\text {min }}=0.980, T_{\text {max }}=0.983$

\section{Refinement}

Refinement on $F^{2}$

Least-squares matrix: full

$R\left[F^{2}>2 \sigma\left(F^{2}\right)\right]=0.064$

$w R\left(F^{2}\right)=0.205$

$S=1.07$

3556 reflections

272 parameters

10 restraints

Primary atom site location: structure-invariant direct methods

Secondary atom site location: difference Fourier map
$F(000)=736$

$D_{\mathrm{x}}=1.160 \mathrm{Mg} \mathrm{m}^{-3}$

Mo $K \alpha$ radiation, $\lambda=0.71073 \AA$

Cell parameters from 7089 reflections

$\theta=2.5-25.3^{\circ}$

$\mu=0.07 \mathrm{~mm}^{-1}$

$T=295 \mathrm{~K}$

Block, colourless

$0.28 \times 0.26 \times 0.24 \mathrm{~mm}$

29557 measured reflections

3556 independent reflections

2130 reflections with $I>2 \sigma(I)$

$R_{\text {int }}=0.046$

$\theta_{\text {max }}=25.4^{\circ}, \theta_{\min }=2.3^{\circ}$

$h=-9 \rightarrow 9$

$k=-11 \rightarrow 11$

$l=-29 \rightarrow 29$

Hydrogen site location: inferred from neighbouring sites

$\mathrm{H}$ atoms treated by a mixture of independent and constrained refinement

$w=1 /\left[\sigma^{2}\left(F_{\mathrm{o}}^{2}\right)+(0.0727 P)^{2}+1.5032 P\right]$

where $P=\left(F_{\mathrm{o}}^{2}+2 F_{\mathrm{c}}^{2}\right) / 3$

$(\Delta / \sigma)_{\max }<0.001$

$\Delta \rho_{\max }=0.44 \mathrm{e}^{-3}$

$\Delta \rho_{\min }=-0.20$ e $\AA^{-3}$

Absolute structure: Flack (1983), 1466 Friedel pairs 


\section{Special details}

Geometry. All s.u.'s (except the s.u. in the dihedral angle between two 1.s. planes) are estimated using the full covariance matrix. The cell s.u.'s are taken into account individually in the estimation of s.u.'s in distances, angles and torsion angles; correlations between s.u.'s in cell parameters are only used when they are defined by crystal symmetry. An approximate (isotropic) treatment of cell s.u.'s is used for estimating s.u.'s involving l.s. planes.

Refinement. Refinement of $F^{2}$ against ALL reflections. The weighted $R$-factor $w R$ and goodness of fit $S$ are based on $F^{2}$, conventional $R$-factors $R$ are based on $F$, with $F$ set to zero for negative $F^{2}$. The threshold expression of $F^{2}>\sigma\left(F^{2}\right)$ is used only for calculating $R$-factors(gt) etc. and is not relevant to the choice of reflections for refinement. $R$-factors based on $F^{2}$ are statistically about twice as large as those based on $F$, and $R$-factors based on ALL data will be even larger.

Fractional atomic coordinates and isotropic or equivalent isotropic displacement parameters $\left(\hat{A}^{2}\right)$

\begin{tabular}{|c|c|c|c|c|c|}
\hline & $x$ & $y$ & $z$ & $U_{\text {iso }} * / U_{\text {eq }}$ & Occ. $(<1)$ \\
\hline $\mathrm{C} 1$ & $0.7266(10)$ & $0.9101(7)$ & $-0.0244(3)$ & $0.084(2)$ & $0.775(9)$ \\
\hline H1A & 0.7411 & 0.8690 & 0.0111 & $0.125^{*}$ & $0.775(9)$ \\
\hline H1B & 0.6538 & 0.9871 & -0.0214 & $0.125^{*}$ & $0.775(9)$ \\
\hline $\mathrm{H} 1 \mathrm{C}$ & 0.8302 & 0.9409 & -0.0380 & $0.125^{*}$ & $0.775(9)$ \\
\hline $\mathrm{C} 2$ & $0.6555(11)$ & $0.8049(8)$ & $-0.0633(4)$ & $0.067(3)$ & $0.775(9)$ \\
\hline $\mathrm{H} 2 \mathrm{~A}$ & 0.5577 & 0.7651 & -0.0475 & $0.080^{*}$ & $0.775(9)$ \\
\hline $\mathrm{H} 2 \mathrm{~B}$ & 0.6260 & 0.8492 & -0.0975 & $0.080^{*}$ & $0.775(9)$ \\
\hline $\mathrm{C} 3$ & $0.9082(10)$ & $0.7205(8)$ & $-0.1156(3)$ & $0.073(2)$ & $0.775(9)$ \\
\hline $\mathrm{H} 3 \mathrm{~A}$ & 0.9375 & 0.8172 & -0.1161 & $0.088^{*}$ & $0.775(9)$ \\
\hline H3B & 1.0047 & 0.6674 & -0.1065 & $0.088^{*}$ & $0.775(9)$ \\
\hline $\mathrm{C} 4$ & $0.8438(10)$ & $0.6778(8)$ & $-0.1703(3)$ & $0.093(3)$ & $0.775(9)$ \\
\hline $\mathrm{H} 4 \mathrm{~A}$ & 0.8211 & 0.5809 & -0.1699 & $0.140^{*}$ & $0.775(9)$ \\
\hline $\mathrm{H} 4 \mathrm{~B}$ & 0.9237 & 0.6975 & -0.1979 & $0.140^{*}$ & $0.775(9)$ \\
\hline $\mathrm{H} 4 \mathrm{C}$ & 0.7454 & 0.7277 & -0.1780 & $0.140^{*}$ & $0.775(9)$ \\
\hline $\mathrm{C} 1 \mathrm{~A}$ & $0.586(4)$ & $0.847(5)$ & -0.0615 (19) & $0.122(16)$ & $0.225(9)$ \\
\hline H1A1 & 0.5521 & 0.9397 & -0.0545 & $0.183^{*}$ & $0.225(9)$ \\
\hline H1A2 & 0.5193 & 0.7847 & -0.0405 & $0.183^{*}$ & $0.225(9)$ \\
\hline H1A3 & 0.5730 & 0.8267 & -0.0998 & $0.183^{*}$ & $0.225(9)$ \\
\hline $\mathrm{C} 2 \mathrm{~A}$ & $0.764(4)$ & $0.8291(15)$ & $-0.0453(9)$ & $0.085(9)$ & $0.225(9)$ \\
\hline $\mathrm{H} 2 \mathrm{~A} 1$ & 0.8345 & 0.8993 & -0.0607 & $0.102 *$ & $0.225(9)$ \\
\hline $\mathrm{H} 2 \mathrm{~A} 2$ & 0.7798 & 0.8221 & -0.0061 & $0.102 *$ & $0.225(9)$ \\
\hline $\mathrm{C} 3 \mathrm{~A}$ & $0.787(3)$ & $0.6858(16)$ & $-0.1341(4)$ & $0.061(7)$ & $0.225(9)$ \\
\hline H3A1 & 0.7019 & 0.7390 & -0.1519 & $0.073^{*}$ & $0.225(9)$ \\
\hline $\mathrm{H} 3 \mathrm{~A} 2$ & 0.7824 & 0.5911 & -0.1467 & $0.073^{*}$ & $0.225(9)$ \\
\hline $\mathrm{C} 4 \mathrm{~A}$ & $0.955(3)$ & $0.749(3)$ & $-0.1425(13)$ & $0.080(9)$ & $0.225(9)$ \\
\hline H4A1 & 0.9478 & 0.8474 & -0.1389 & $0.120^{*}$ & $0.225(9)$ \\
\hline $\mathrm{H} 4 \mathrm{~A} 2$ & 0.9944 & 0.7263 & -0.1783 & $0.120^{*}$ & $0.225(9)$ \\
\hline H4A3 & 1.0288 & 0.7136 & -0.1154 & $0.120^{*}$ & $0.225(9)$ \\
\hline $\mathrm{C} 5$ & $0.7811(6)$ & $0.5793(4)$ & $-0.04177(17)$ & $0.0666(12)$ & \\
\hline C6 & $0.8764(6)$ & $0.4644(5)$ & $-0.05697(19)$ & $0.0725(13)$ & \\
\hline H6 & 0.9326 & 0.4651 & -0.0901 & $0.087^{*}$ & \\
\hline $\mathrm{C} 7$ & $0.8865(6)$ & $0.3522(5)$ & $-0.02341(19)$ & $0.0680(12)$ & \\
\hline H7 & 0.9509 & 0.2782 & -0.0342 & $0.082^{*}$ & \\
\hline $\mathrm{C} 8$ & $0.8054(5)$ & $0.3444(4)$ & $0.02559(16)$ & $0.0541(10)$ & \\
\hline C9 & $0.7068(6)$ & $0.4550(5)$ & $0.03971(16)$ & $0.0613(11)$ & \\
\hline
\end{tabular}




$\begin{array}{lllll}\text { C10 } & 0.6941(6) & 0.5712(4) & 0.00699(17) & 0.0662(12) \\ \text { H10 } & 0.6274 & 0.6438 & 0.0177 & 0.079^{*} \\ \text { C11 } & 0.8246(6) & 0.2245(5) & 0.06113(19) & 0.0643(12) \\ \text { H11 } & 0.8909 & 0.1522 & 0.0499 & 0.077^{*} \\ \text { C12 } & 0.7727(5) & 0.1017(4) & 0.14256(17) & 0.0574(11) \\ \text { C13 } & 0.8522(6) & -0.0207(5) & 0.12960(18) & 0.0706(13) \\ \text { H13 } & 0.9012 & -0.0306 & 0.0955 & 0.085^{*} \\ \text { C14 } & 0.8591(6) & -0.1287(5) & 0.16721(18) & 0.0679(12) \\ \text { H14 } & 0.9130 & -0.2094 & 0.1577 & 0.082^{*} \\ \text { C15 } & 0.7872(5) & -0.1183(4) & 0.21878(16) & 0.0515(10) \\ \text { C16 } & 0.7109(5) & 0.0078(4) & 0.23007(17) & 0.0616(11) \\ \text { H16 } & 0.6638 & 0.0210 & 0.2643 & 0.074^{*} \\ \text { C17 } & 0.7033(5) & 0.1122(5) & 0.19253(18) & 0.0622(11) \\ \text { H17 } & 0.6487 & 0.1929 & 0.2017 & 0.075^{*} \\ \text { C18 } & 0.8789(6) & -0.3514(5) & 0.2453(2) & 0.0726(13) \\ \text { H18A } & 0.9128 & -0.3912 & 0.2799 & 0.087^{*} \\ \text { H18B } & 0.9765 & -0.3306 & 0.2244 & 0.087^{*} \\ \text { C19 } & 0.7787(9) & -0.4567(6) & 0.2141(2) & 0.109(2) \\ \text { H19A } & 0.6855 & -0.4825 & 0.2356 & 0.164^{*} \\ \text { H19B } & 0.8443 & -0.5366 & 0.2070 & 0.164^{*} \\ \text { H19C } & 0.7429 & -0.4176 & 0.1801 & 0.164^{*} \\ \text { C20 } & 0.7125(6) & -0.2143(5) & 0.3091(2) & 0.0764(14) \\ \text { H20A } & 0.6822 & -0.3058 & 0.3213 & 0.092^{*} \\ \text { H20B } & 0.6132 & -0.1607 & 0.3054 & 0.092^{*} \\ \text { C21 } & 0.8191(9) & -0.1487(7) & 0.3515(2) & 0.113(2) \\ \text { H21A } & 0.9208 & -0.1975 & 0.3535 & 0.170^{*} \\ \text { H21B } & 0.7657 & -0.1521 & 0.3864 & 0.170^{*} \\ \text { H21C } & 0.8393 & -0.0546 & 0.3417 & 0.170^{*} \\ \text { N1 } & 0.7767(7) & 0.6954(4) & -0.07378(16) & 0.1047(19) \\ \text { N2 } & 0.7542(4) & 0.2174(4) & 0.10594(14) & 0.0626(10) \\ \text { N3 } & 0.7918(5) & -0.2243(4) & 0.25593(14) & 0.0654(10) \\ \text { O1 } & 0.6208(5) & 0.4508(4) & 0.08624(14) & 0.0858(11) \\ \text { H1 } & 0.643(8) & 0.379(4) & 0.105(2) & 0.129^{*} \\ & & & & \end{array}$

Atomic displacement parameters $\left(\AA^{2}\right)$

\begin{tabular}{lllllll}
\hline & $U^{11}$ & $U^{22}$ & $U^{33}$ & $U^{12}$ & $U^{13}$ & $U^{23}$ \\
\hline C1 & $0.091(5)$ & $0.071(5)$ & $0.089(5)$ & $0.010(4)$ & $0.006(4)$ & $-0.013(4)$ \\
C2 & $0.084(8)$ & $0.057(5)$ & $0.059(4)$ & $0.014(5)$ & $-0.005(5)$ & $0.004(3)$ \\
C3 & $0.081(6)$ & $0.061(4)$ & $0.078(6)$ & $-0.007(4)$ & $0.005(4)$ & $0.004(4)$ \\
C4 & $0.099(6)$ & $0.105(6)$ & $0.075(5)$ & $0.017(5)$ & $0.000(4)$ & $-0.008(4)$ \\
C1A & $0.11(3)$ & $0.11(3)$ & $0.15(3)$ & $-0.01(2)$ & $0.04(3)$ & $0.01(3)$ \\
C2A & $0.12(3)$ & $0.08(2)$ & $0.058(16)$ & $-0.004(18)$ & $-0.006(17)$ & $0.015(14)$ \\
C3A & $0.13(2)$ & $0.021(8)$ & $0.036(11)$ & $0.008(11)$ & $0.021(12)$ & $0.000(7)$ \\
C4A & $0.076(17)$ & $0.080(19)$ & $0.09(2)$ & $-0.020(14)$ & $0.023(15)$ & $-0.010(16)$ \\
C5 & $0.091(3)$ & $0.054(3)$ & $0.055(2)$ & $0.012(3)$ & $0.019(3)$ & $0.003(2)$ \\
C6 & $0.090(4)$ & $0.061(3)$ & $0.067(3)$ & $0.008(3)$ & $0.018(3)$ & $-0.006(2)$ \\
C7 & $0.075(3)$ & $0.054(3)$ & $0.075(3)$ & $0.010(2)$ & $0.004(3)$ & $-0.005(2)$
\end{tabular}


supporting information

$\begin{array}{lllllll}\text { C8 } & 0.050(2) & 0.048(2) & 0.064(3) & 0.000(2) & -0.010(2) & 0.0036(19) \\ \text { C9 } & 0.059(2) & 0.071(3) & 0.054(2) & -0.004(2) & 0.0005(17) & 0.001(2) \\ \text { C10 } & 0.081(3) & 0.059(3) & 0.059(2) & 0.017(3) & 0.015(2) & 0.005(2) \\ \text { C11 } & 0.056(3) & 0.071(3) & 0.065(3) & -0.002(2) & -0.012(2) & -0.003(2) \\ \text { C12 } & 0.052(2) & 0.049(2) & 0.071(3) & 0.000(2) & -0.017(2) & 0.010(2) \\ \text { C13 } & 0.081(3) & 0.080(3) & 0.050(2) & -0.005(3) & -0.002(2) & 0.002(2) \\ \text { C14 } & 0.078(3) & 0.061(3) & 0.065(3) & 0.013(2) & -0.007(2) & 0.006(2) \\ \text { C15 } & 0.051(2) & 0.046(2) & 0.058(2) & -0.002(2) & -0.011(2) & 0.0053(19) \\ \text { C16 } & 0.056(2) & 0.062(3) & 0.067(3) & 0.001(2) & -0.003(2) & 0.004(2) \\ \text { C17 } & 0.056(3) & 0.064(3) & 0.067(3) & 0.001(2) & -0.006(2) & 0.002(2) \\ \text { C18 } & 0.077(3) & 0.063(3) & 0.077(3) & 0.015(3) & -0.004(3) & 0.014(3) \\ \text { C19 } & 0.144(6) & 0.066(3) & 0.116(4) & 0.001(4) & -0.014(5) & -0.014(3) \\ \text { C20 } & 0.073(3) & 0.063(3) & 0.093(3) & -0.001(3) & 0.001(3) & 0.018(3) \\ \text { C21 } & 0.145(6) & 0.123(5) & 0.072(3) & -0.005(5) & -0.001(4) & -0.008(3) \\ \text { N1 } & 0.176(5) & 0.065(3) & 0.072(3) & 0.038(3) & 0.061(3) & 0.020(2) \\ \text { N2 } & 0.061(2) & 0.065(2) & 0.061(2) & 0.0032(19) & -0.0139(19) & -0.0030(18) \\ \text { N3 } & 0.081(2) & 0.054(2) & 0.062(2) & 0.011(2) & 0.000(2) & 0.0053(17) \\ \text { O1 } & 0.105(3) & 0.084(2) & 0.068(2) & 0.028(2) & 0.0249(18) & 0.0131(18) \\ & & & & & & \end{array}$

Geometric parameters $\left(\AA,{ }^{\circ}\right)$

\begin{tabular}{llll}
\hline $\mathrm{C} 1-\mathrm{C} 2$ & $1.513(8)$ & $\mathrm{C} 8-\mathrm{C} 9$ & $1.388(6)$ \\
$\mathrm{C} 1-\mathrm{H} 1 \mathrm{~A}$ & 0.9600 & $\mathrm{C} 8-\mathrm{C} 11$ & $1.461(6)$ \\
$\mathrm{C} 1-\mathrm{H} 1 \mathrm{~B}$ & 0.9600 & $\mathrm{C} 9-\mathrm{O} 1$ & $1.338(5)$ \\
$\mathrm{C} 1-\mathrm{H} 1 \mathrm{C}$ & 0.9600 & $\mathrm{C} 9-\mathrm{C} 10$ & $1.387(6)$ \\
$\mathrm{C} 2-\mathrm{N} 1$ & $1.478(7)$ & $\mathrm{C} 10-\mathrm{H} 10$ & 0.9300 \\
$\mathrm{C} 2-\mathrm{H} 2 \mathrm{~A}$ & 0.9700 & $\mathrm{C} 11-\mathrm{N} 2$ & $1.239(5)$ \\
$\mathrm{C} 2-\mathrm{H} 2 \mathrm{~B}$ & 0.9700 & $\mathrm{C} 11-\mathrm{H} 11$ & 0.9300 \\
$\mathrm{C} 3-\mathrm{C} 4$ & $1.495(7)$ & $\mathrm{C} 12-\mathrm{C} 17$ & $1.350(6)$ \\
$\mathrm{C} 3-\mathrm{N} 1$ & $1.504(7)$ & $\mathrm{C} 12-\mathrm{C} 13$ & $1.392(6)$ \\
$\mathrm{C} 3-\mathrm{H} 3 \mathrm{~A}$ & 0.9700 & $\mathrm{C} 12-\mathrm{N} 2$ & $1.444(5)$ \\
$\mathrm{C} 3-\mathrm{H} 3 \mathrm{~B}$ & 0.9700 & $\mathrm{C} 13-\mathrm{C} 14$ & $1.395(6)$ \\
$\mathrm{C} 4-\mathrm{H} 4 \mathrm{~A}$ & 0.9600 & $\mathrm{C} 13-\mathrm{H} 13$ & 0.9300 \\
$\mathrm{C} 4-\mathrm{H} 4 \mathrm{~B}$ & 0.9600 & $\mathrm{C} 14-\mathrm{C} 15$ & $1.394(6)$ \\
$\mathrm{C} 4-\mathrm{H} 4 \mathrm{C}$ & 0.9600 & $\mathrm{C} 14-\mathrm{H} 14$ & 0.9300 \\
$\mathrm{C} 1 \mathrm{~A}-\mathrm{C} 2 \mathrm{~A}$ & $1.523(10)$ & $\mathrm{C} 15-\mathrm{N} 3$ & $1.373(5)$ \\
$\mathrm{C} 1 \mathrm{~A}-\mathrm{H} 1 \mathrm{~A} 1$ & 0.9600 & $\mathrm{C} 15-\mathrm{C} 16$ & $1.403(6)$ \\
$\mathrm{C} 1 \mathrm{~A}-\mathrm{H} 1 \mathrm{~A} 2$ & 0.9600 & $\mathrm{C} 16-\mathrm{C} 17$ & $1.368(6)$ \\
$\mathrm{C} 1 \mathrm{~A}-\mathrm{H} 1 \mathrm{~A} 3$ & 0.9600 & $\mathrm{C} 16-\mathrm{H} 16$ & 0.9300 \\
$\mathrm{C} 2 \mathrm{~A}-\mathrm{N} 1$ & $1.477(10)$ & $\mathrm{C} 17-\mathrm{H} 17$ & 0.9300 \\
$\mathrm{C} 2 \mathrm{~A}-\mathrm{H} 2 \mathrm{~A} 1$ & $\mathrm{C} 18-\mathrm{N} 3$ & $1.450(5)$ \\
$\mathrm{C} 2 \mathrm{~A}-\mathrm{H} 2 \mathrm{~A} 2$ & 0.9700 & $\mathrm{C} 18-\mathrm{C} 19$ & $1.516(7)$ \\
$\mathrm{C} 3 \mathrm{~A}-\mathrm{N} 1$ & 0.9700 & $\mathrm{C} 18-\mathrm{H} 18 \mathrm{~A}$ & 0.9700 \\
$\mathrm{C} 3 \mathrm{~A}-\mathrm{C} 4 \mathrm{~A}$ & $1.478(9)$ & $\mathrm{C} 18-\mathrm{H} 18 \mathrm{~B}$ & 0.9700 \\
$\mathrm{C} 3 \mathrm{~A}-\mathrm{H} 3 \mathrm{~A} 1$ & $1.524(10)$ & $\mathrm{C} 19-\mathrm{H} 19 \mathrm{~A}$ & 0.9600 \\
$\mathrm{C} 3 \mathrm{~A}-\mathrm{H} 3 \mathrm{~A} 2$ & 0.9700 & $\mathrm{C} 19-\mathrm{H} 19 \mathrm{~B}$ & 0.9600 \\
$\mathrm{C} 4 \mathrm{~A}-\mathrm{H} 4 \mathrm{~A} 1$ & $\mathrm{C} 19-\mathrm{H} 19 \mathrm{C}$ & 0.9600 \\
$\mathrm{C} 4 \mathrm{~A}-\mathrm{H} 4 \mathrm{~A} 2$ & 0.9700 & $\mathrm{C} 20-\mathrm{N} 3$ & $1.455(6)$ \\
& 0.9600 & &
\end{tabular}




\begin{tabular}{|c|c|c|c|}
\hline $\mathrm{C} 4 \mathrm{~A}-\mathrm{H} 4 \mathrm{~A} 3$ & 0.9600 & $\mathrm{C} 20-\mathrm{C} 21$ & $1.497(7)$ \\
\hline $\mathrm{C} 5-\mathrm{N} 1$ & $1.372(5)$ & $\mathrm{C} 20-\mathrm{H} 20 \mathrm{~A}$ & 0.9700 \\
\hline $\mathrm{C} 5-\mathrm{C} 10$ & $1.390(6)$ & $\mathrm{C} 20-\mathrm{H} 20 \mathrm{~B}$ & 0.9700 \\
\hline $\mathrm{C} 5-\mathrm{C} 6$ & $1.412(6)$ & $\mathrm{C} 21-\mathrm{H} 21 \mathrm{~A}$ & 0.9600 \\
\hline $\mathrm{C} 6-\mathrm{C} 7$ & $1.366(6)$ & $\mathrm{C} 21-\mathrm{H} 21 \mathrm{~B}$ & 0.9600 \\
\hline $\mathrm{C} 6-\mathrm{H} 6$ & 0.9300 & $\mathrm{C} 21-\mathrm{H} 21 \mathrm{C}$ & 0.9600 \\
\hline $\mathrm{C} 7-\mathrm{C} 8$ & $1.371(6)$ & $\mathrm{O} 1-\mathrm{H} 1$ & $0.86(2)$ \\
\hline $\mathrm{C} 7-\mathrm{H} 7$ & 0.9300 & & \\
\hline $\mathrm{N} 1-\mathrm{C} 2-\mathrm{C} 1$ & $109.6(7)$ & $\mathrm{C} 17-\mathrm{C} 12-\mathrm{C} 13$ & $117.8(4)$ \\
\hline $\mathrm{N} 1-\mathrm{C} 2-\mathrm{H} 2 \mathrm{~A}$ & 109.8 & $\mathrm{C} 17-\mathrm{C} 12-\mathrm{N} 2$ & $117.2(4)$ \\
\hline $\mathrm{C} 1-\mathrm{C} 2-\mathrm{H} 2 \mathrm{~A}$ & 109.8 & $\mathrm{C} 13-\mathrm{C} 12-\mathrm{N} 2$ & $125.0(4)$ \\
\hline $\mathrm{N} 1-\mathrm{C} 2-\mathrm{H} 2 \mathrm{~B}$ & 109.8 & $\mathrm{C} 12-\mathrm{C} 13-\mathrm{C} 14$ & $120.8(4)$ \\
\hline $\mathrm{C} 1-\mathrm{C} 2-\mathrm{H} 2 \mathrm{~B}$ & 109.8 & $\mathrm{C} 12-\mathrm{C} 13-\mathrm{H} 13$ & 119.6 \\
\hline $\mathrm{H} 2 \mathrm{~A}-\mathrm{C} 2-\mathrm{H} 2 \mathrm{~B}$ & 108.2 & $\mathrm{C} 14-\mathrm{C} 13-\mathrm{H} 13$ & 119.6 \\
\hline $\mathrm{C} 4-\mathrm{C} 3-\mathrm{N} 1$ & $107.9(7)$ & $\mathrm{C} 15-\mathrm{C} 14-\mathrm{C} 13$ & $121.5(4)$ \\
\hline $\mathrm{C} 4-\mathrm{C} 3-\mathrm{H} 3 \mathrm{~A}$ & 110.1 & $\mathrm{C} 15-\mathrm{C} 14-\mathrm{H} 14$ & 119.2 \\
\hline $\mathrm{N} 1-\mathrm{C} 3-\mathrm{H} 3 \mathrm{~A}$ & 110.1 & $\mathrm{C} 13-\mathrm{C} 14-\mathrm{H} 14$ & 119.2 \\
\hline $\mathrm{C} 4-\mathrm{C} 3-\mathrm{H} 3 \mathrm{~B}$ & 110.1 & $\mathrm{~N} 3-\mathrm{C} 15-\mathrm{C} 14$ & $122.1(4)$ \\
\hline $\mathrm{N} 1-\mathrm{C} 3-\mathrm{H} 3 \mathrm{~B}$ & 110.1 & $\mathrm{~N} 3-\mathrm{C} 15-\mathrm{C} 16$ & $122.5(4)$ \\
\hline $\mathrm{H} 3 \mathrm{~A}-\mathrm{C} 3-\mathrm{H} 3 \mathrm{~B}$ & 108.4 & $\mathrm{C} 14-\mathrm{C} 15-\mathrm{C} 16$ & $115.4(4)$ \\
\hline $\mathrm{C} 2 \mathrm{~A}-\mathrm{C} 1 \mathrm{~A}-\mathrm{H} 1 \mathrm{~A} 1$ & 109.5 & $\mathrm{C} 17-\mathrm{C} 16-\mathrm{C} 15$ & $122.4(4)$ \\
\hline $\mathrm{C} 2 \mathrm{~A}-\mathrm{C} 1 \mathrm{~A}-\mathrm{H} 1 \mathrm{~A} 2$ & 109.5 & $\mathrm{C} 17-\mathrm{C} 16-\mathrm{H} 16$ & 118.8 \\
\hline $\mathrm{H} 1 \mathrm{~A} 1-\mathrm{C} 1 \mathrm{~A}-\mathrm{H} 1 \mathrm{~A} 2$ & 109.5 & $\mathrm{C} 15-\mathrm{C} 16-\mathrm{H} 16$ & 118.8 \\
\hline $\mathrm{C} 2 \mathrm{~A}-\mathrm{C} 1 \mathrm{~A}-\mathrm{H} 1 \mathrm{~A} 3$ & 109.5 & $\mathrm{C} 12-\mathrm{C} 17-\mathrm{C} 16$ & $122.0(4)$ \\
\hline $\mathrm{H} 1 \mathrm{~A} 1-\mathrm{C} 1 \mathrm{~A}-\mathrm{H} 1 \mathrm{~A} 3$ & 109.5 & $\mathrm{C} 12-\mathrm{C} 17-\mathrm{H} 17$ & 119.0 \\
\hline $\mathrm{H} 1 \mathrm{~A} 2-\mathrm{C} 1 \mathrm{~A}-\mathrm{H} 1 \mathrm{~A} 3$ & 109.5 & $\mathrm{C} 16-\mathrm{C} 17-\mathrm{H} 17$ & 119.0 \\
\hline $\mathrm{N} 1-\mathrm{C} 2 \mathrm{~A}-\mathrm{C} 1 \mathrm{~A}$ & $93(2)$ & N3-C18-C19 & $113.4(4)$ \\
\hline $\mathrm{N} 1-\mathrm{C} 2 \mathrm{~A}-\mathrm{H} 2 \mathrm{~A} 1$ & 113.2 & $\mathrm{~N} 3-\mathrm{C} 18-\mathrm{H} 18 \mathrm{~A}$ & 108.9 \\
\hline $\mathrm{C} 1 \mathrm{~A}-\mathrm{C} 2 \mathrm{~A}-\mathrm{H} 2 \mathrm{~A} 1$ & 113.2 & $\mathrm{C} 19-\mathrm{C} 18-\mathrm{H} 18 \mathrm{~A}$ & 108.9 \\
\hline $\mathrm{N} 1-\mathrm{C} 2 \mathrm{~A}-\mathrm{H} 2 \mathrm{~A} 2$ & 113.2 & $\mathrm{~N} 3-\mathrm{C} 18-\mathrm{H} 18 \mathrm{~B}$ & 108.9 \\
\hline $\mathrm{C} 1 \mathrm{~A}-\mathrm{C} 2 \mathrm{~A}-\mathrm{H} 2 \mathrm{~A} 2$ & 113.2 & $\mathrm{C} 19-\mathrm{C} 18-\mathrm{H} 18 \mathrm{~B}$ & 108.9 \\
\hline $\mathrm{H} 2 \mathrm{~A} 1-\mathrm{C} 2 \mathrm{~A}-\mathrm{H} 2 \mathrm{~A} 2$ & 110.5 & $\mathrm{H} 18 \mathrm{~A}-\mathrm{C} 18-\mathrm{H} 18 \mathrm{~B}$ & 107.7 \\
\hline $\mathrm{N} 1-\mathrm{C} 3 \mathrm{~A}-\mathrm{C} 4 \mathrm{~A}$ & $99.1(15)$ & $\mathrm{C} 18-\mathrm{C} 19-\mathrm{H} 19 \mathrm{~A}$ & 109.5 \\
\hline $\mathrm{N} 1-\mathrm{C} 3 \mathrm{~A}-\mathrm{H} 3 \mathrm{~A} 1$ & 111.9 & $\mathrm{C} 18-\mathrm{C} 19-\mathrm{H} 19 \mathrm{~B}$ & 109.5 \\
\hline $\mathrm{C} 4 \mathrm{~A}-\mathrm{C} 3 \mathrm{~A}-\mathrm{H} 3 \mathrm{~A} 1$ & 111.9 & $\mathrm{H} 19 \mathrm{~A}-\mathrm{C} 19-\mathrm{H} 19 \mathrm{~B}$ & 109.5 \\
\hline $\mathrm{N} 1-\mathrm{C} 3 \mathrm{~A}-\mathrm{H} 3 \mathrm{~A} 2$ & 111.9 & $\mathrm{C} 18-\mathrm{C} 19-\mathrm{H} 19 \mathrm{C}$ & 109.5 \\
\hline $\mathrm{C} 4 \mathrm{~A}-\mathrm{C} 3 \mathrm{~A}-\mathrm{H} 3 \mathrm{~A} 2$ & 111.9 & $\mathrm{H} 19 \mathrm{~A}-\mathrm{C} 19-\mathrm{H} 19 \mathrm{C}$ & 109.5 \\
\hline $\mathrm{H} 3 \mathrm{~A} 1-\mathrm{C} 3 \mathrm{~A}-\mathrm{H} 3 \mathrm{~A} 2$ & 109.6 & $\mathrm{H} 19 \mathrm{~B}-\mathrm{C} 19-\mathrm{H} 19 \mathrm{C}$ & 109.5 \\
\hline $\mathrm{C} 3 \mathrm{~A}-\mathrm{C} 4 \mathrm{~A}-\mathrm{H} 4 \mathrm{~A} 1$ & 109.5 & $\mathrm{~N} 3-\mathrm{C} 20-\mathrm{C} 21$ & $112.6(4)$ \\
\hline $\mathrm{C} 3 \mathrm{~A}-\mathrm{C} 4 \mathrm{~A}-\mathrm{H} 4 \mathrm{~A} 2$ & 109.5 & $\mathrm{~N} 3-\mathrm{C} 20-\mathrm{H} 20 \mathrm{~A}$ & 109.1 \\
\hline $\mathrm{H} 4 \mathrm{~A} 1-\mathrm{C} 4 \mathrm{~A}-\mathrm{H} 4 \mathrm{~A} 2$ & 109.5 & $\mathrm{C} 21-\mathrm{C} 20-\mathrm{H} 20 \mathrm{~A}$ & 109.1 \\
\hline $\mathrm{C} 3 \mathrm{~A}-\mathrm{C} 4 \mathrm{~A}-\mathrm{H} 4 \mathrm{~A} 3$ & 109.5 & $\mathrm{~N} 3-\mathrm{C} 20-\mathrm{H} 20 \mathrm{~B}$ & 109.1 \\
\hline $\mathrm{H} 4 \mathrm{~A} 1-\mathrm{C} 4 \mathrm{~A}-\mathrm{H} 4 \mathrm{~A} 3$ & 109.5 & $\mathrm{C} 21-\mathrm{C} 20-\mathrm{H} 20 \mathrm{~B}$ & 109.1 \\
\hline $\mathrm{H} 4 \mathrm{~A} 2-\mathrm{C} 4 \mathrm{~A}-\mathrm{H} 4 \mathrm{~A} 3$ & 109.5 & $\mathrm{H} 20 \mathrm{~A}-\mathrm{C} 20-\mathrm{H} 20 \mathrm{~B}$ & 107.8 \\
\hline $\mathrm{N} 1-\mathrm{C} 5-\mathrm{C} 10$ & $121.4(4)$ & $\mathrm{C} 20-\mathrm{C} 21-\mathrm{H} 21 \mathrm{~A}$ & 109.5 \\
\hline $\mathrm{N} 1-\mathrm{C} 5-\mathrm{C} 6$ & $120.9(4)$ & $\mathrm{C} 20-\mathrm{C} 21-\mathrm{H} 21 \mathrm{~B}$ & 109.5 \\
\hline $\mathrm{C} 10-\mathrm{C} 5-\mathrm{C} 6$ & $117.7(4)$ & $\mathrm{H} 21 \mathrm{~A}-\mathrm{C} 21-\mathrm{H} 21 \mathrm{~B}$ & 109.5 \\
\hline
\end{tabular}




\begin{tabular}{|c|c|c|c|}
\hline $\mathrm{C} 7-\mathrm{C} 6-\mathrm{C} 5$ & $120.4(4)$ & $\mathrm{C} 20-\mathrm{C} 21-\mathrm{H} 21 \mathrm{C}$ & 109.5 \\
\hline $\mathrm{C} 7-\mathrm{C} 6-\mathrm{H} 6$ & 119.8 & $\mathrm{H} 21 \mathrm{~A}-\mathrm{C} 21-\mathrm{H} 21 \mathrm{C}$ & 109.5 \\
\hline $\mathrm{C} 5-\mathrm{C} 6-\mathrm{H} 6$ & 119.8 & $\mathrm{H} 21 \mathrm{~B}-\mathrm{C} 21-\mathrm{H} 21 \mathrm{C}$ & 109.5 \\
\hline $\mathrm{C} 6-\mathrm{C} 7-\mathrm{C} 8$ & $122.6(4)$ & $\mathrm{C} 5-\mathrm{N} 1-\mathrm{C} 2 \mathrm{~A}$ & $117.2(10)$ \\
\hline $\mathrm{C} 6-\mathrm{C} 7-\mathrm{H} 7$ & 118.7 & $\mathrm{C} 5-\mathrm{N} 1-\mathrm{C} 3 \mathrm{~A}$ & $120.9(7)$ \\
\hline $\mathrm{C} 8-\mathrm{C} 7-\mathrm{H} 7$ & 118.7 & $\mathrm{C} 2 \mathrm{~A}-\mathrm{N} 1-\mathrm{C} 3 \mathrm{~A}$ & $121.9(12)$ \\
\hline $\mathrm{C} 7-\mathrm{C} 8-\mathrm{C} 9$ & $117.2(4)$ & $\mathrm{C} 5-\mathrm{N} 1-\mathrm{C} 2$ & $120.7(5)$ \\
\hline $\mathrm{C} 7-\mathrm{C} 8-\mathrm{C} 11$ & $120.7(4)$ & $\mathrm{C} 2 \mathrm{~A}-\mathrm{N} 1-\mathrm{C} 2$ & $40.3(12)$ \\
\hline $\mathrm{C} 9-\mathrm{C} 8-\mathrm{C} 11$ & $122.1(4)$ & $\mathrm{C} 3 \mathrm{~A}-\mathrm{N} 1-\mathrm{C} 2$ & $104.8(9)$ \\
\hline $\mathrm{O} 1-\mathrm{C} 9-\mathrm{C} 10$ & $118.3(4)$ & $\mathrm{C} 5-\mathrm{N} 1-\mathrm{C} 3$ & $120.0(5)$ \\
\hline $\mathrm{O} 1-\mathrm{C} 9-\mathrm{C} 8$ & $119.7(4)$ & $\mathrm{C} 2 \mathrm{~A}-\mathrm{N} 1-\mathrm{C} 3$ & $103.2(12)$ \\
\hline $\mathrm{C} 10-\mathrm{C} 9-\mathrm{C} 8$ & $122.0(4)$ & $\mathrm{C} 3 \mathrm{~A}-\mathrm{N} 1-\mathrm{C} 3$ & $45.1(8)$ \\
\hline $\mathrm{C} 9-\mathrm{C} 10-\mathrm{C} 5$ & $120.1(4)$ & $\mathrm{C} 2-\mathrm{N} 1-\mathrm{C} 3$ & $118.8(6)$ \\
\hline $\mathrm{C} 9-\mathrm{C} 10-\mathrm{H} 10$ & 120.0 & $\mathrm{C} 11-\mathrm{N} 2-\mathrm{C} 12$ & $122.8(4)$ \\
\hline $\mathrm{C} 5-\mathrm{C} 10-\mathrm{H} 10$ & 120.0 & $\mathrm{C} 15-\mathrm{N} 3-\mathrm{C} 18$ & $122.3(4)$ \\
\hline $\mathrm{N} 2-\mathrm{C} 11-\mathrm{C} 8$ & $121.3(4)$ & $\mathrm{C} 15-\mathrm{N} 3-\mathrm{C} 20$ & $121.8(4)$ \\
\hline $\mathrm{N} 2-\mathrm{C} 11-\mathrm{H} 11$ & 119.4 & $\mathrm{C} 18-\mathrm{N} 3-\mathrm{C} 20$ & $115.9(4)$ \\
\hline $\mathrm{C} 8-\mathrm{C} 11-\mathrm{H} 11$ & 119.4 & $\mathrm{C} 9-\mathrm{O} 1-\mathrm{H} 1$ & $112(4)$ \\
\hline $\mathrm{N} 1-\mathrm{C} 5-\mathrm{C} 6-\mathrm{C} 7$ & $176.6(5)$ & $\mathrm{C} 6-\mathrm{C} 5-\mathrm{N} 1-\mathrm{C} 2$ & $169.0(6)$ \\
\hline $\mathrm{C} 10-\mathrm{C} 5-\mathrm{C} 6-\mathrm{C} 7$ & $-2.5(8)$ & $\mathrm{C} 10-\mathrm{C} 5-\mathrm{N} 1-\mathrm{C} 3$ & $160.6(5)$ \\
\hline $\mathrm{C} 5-\mathrm{C} 6-\mathrm{C} 7-\mathrm{C} 8$ & $0.6(8)$ & $\mathrm{C} 6-\mathrm{C} 5-\mathrm{N} 1-\mathrm{C} 3$ & $-18.5(8)$ \\
\hline $\mathrm{C} 6-\mathrm{C} 7-\mathrm{C} 8-\mathrm{C} 9$ & $1.8(7)$ & $\mathrm{C} 1 \mathrm{~A}-\mathrm{C} 2 \mathrm{~A}-\mathrm{N} 1-\mathrm{C} 5$ & $-106(2)$ \\
\hline $\mathrm{C} 6-\mathrm{C} 7-\mathrm{C} 8-\mathrm{C} 11$ & $-177.7(4)$ & $\mathrm{C} 1 \mathrm{~A}-\mathrm{C} 2 \mathrm{~A}-\mathrm{N} 1-\mathrm{C} 3 \mathrm{~A}$ & $75(3)$ \\
\hline $\mathrm{C} 7-\mathrm{C} 8-\mathrm{C} 9-\mathrm{O} 1$ & $177.9(4)$ & $\mathrm{C} 1 \mathrm{~A}-\mathrm{C} 2 \mathrm{~A}-\mathrm{N} 1-\mathrm{C} 2$ & $0(2)$ \\
\hline $\mathrm{C} 11-\mathrm{C} 8-\mathrm{C} 9-\mathrm{O} 1$ & $-2.7(6)$ & $\mathrm{C} 1 \mathrm{~A}-\mathrm{C} 2 \mathrm{~A}-\mathrm{N} 1-\mathrm{C} 3$ & $120(2)$ \\
\hline $\mathrm{C} 7-\mathrm{C} 8-\mathrm{C} 9-\mathrm{C} 10$ & $-2.2(6)$ & $\mathrm{C} 4 \mathrm{~A}-\mathrm{C} 3 \mathrm{~A}-\mathrm{N} 1-\mathrm{C} 5$ & $-110.9(14)$ \\
\hline $\mathrm{C} 11-\mathrm{C} 8-\mathrm{C} 9-\mathrm{C} 10$ & $177.2(4)$ & $\mathrm{C} 4 \mathrm{~A}-\mathrm{C} 3 \mathrm{~A}-\mathrm{N} 1-\mathrm{C} 2 \mathrm{~A}$ & $68(2)$ \\
\hline $\mathrm{O} 1-\mathrm{C} 9-\mathrm{C} 10-\mathrm{C} 5$ & $-179.8(4)$ & $\mathrm{C} 4 \mathrm{~A}-\mathrm{C} 3 \mathrm{~A}-\mathrm{N} 1-\mathrm{C} 2$ & $108.3(15)$ \\
\hline $\mathrm{C} 8-\mathrm{C} 9-\mathrm{C} 10-\mathrm{C} 5$ & $0.3(7)$ & $\mathrm{C} 4 \mathrm{~A}-\mathrm{C} 3 \mathrm{~A}-\mathrm{N} 1-\mathrm{C} 3$ & $-7.8(14)$ \\
\hline $\mathrm{N} 1-\mathrm{C} 5-\mathrm{C} 10-\mathrm{C} 9$ & $-177.1(5)$ & $\mathrm{C} 1-\mathrm{C} 2-\mathrm{N} 1-\mathrm{C} 5$ & $91.1(9)$ \\
\hline $\mathrm{C} 6-\mathrm{C} 5-\mathrm{C} 10-\mathrm{C} 9$ & $2.1(7)$ & $\mathrm{C} 1-\mathrm{C} 2-\mathrm{N} 1-\mathrm{C} 2 \mathrm{~A}$ & $-5.8(16)$ \\
\hline $\mathrm{C} 7-\mathrm{C} 8-\mathrm{C} 11-\mathrm{N} 2$ & $178.7(4)$ & $\mathrm{C} 1-\mathrm{C} 2-\mathrm{N} 1-\mathrm{C} 3 \mathrm{~A}$ & $-128.1(9)$ \\
\hline $\mathrm{C} 9-\mathrm{C} 8-\mathrm{C} 11-\mathrm{N} 2$ & $-0.8(6)$ & $\mathrm{C} 1-\mathrm{C} 2-\mathrm{N} 1-\mathrm{C} 3$ & $-81.6(8)$ \\
\hline $\mathrm{C} 17-\mathrm{C} 12-\mathrm{C} 13-\mathrm{C} 14$ & $0.2(6)$ & $\mathrm{C} 4-\mathrm{C} 3-\mathrm{N} 1-\mathrm{C} 5$ & $99.5(7)$ \\
\hline $\mathrm{N} 2-\mathrm{C} 12-\mathrm{C} 13-\mathrm{C} 14$ & $-177.9(4)$ & $\mathrm{C} 4-\mathrm{C} 3-\mathrm{N} 1-\mathrm{C} 2 \mathrm{~A}$ & $-127.9(12)$ \\
\hline $\mathrm{C} 12-\mathrm{C} 13-\mathrm{C} 14-\mathrm{C} 15$ & $0.0(7)$ & $\mathrm{C} 4-\mathrm{C} 3-\mathrm{N} 1-\mathrm{C} 3 \mathrm{~A}$ & $-5.7(10)$ \\
\hline $\mathrm{C} 13-\mathrm{C} 14-\mathrm{C} 15-\mathrm{N} 3$ & $179.3(4)$ & $\mathrm{C} 4-\mathrm{C} 3-\mathrm{N} 1-\mathrm{C} 2$ & $-87.8(7)$ \\
\hline $\mathrm{C} 13-\mathrm{C} 14-\mathrm{C} 15-\mathrm{C} 16$ & $-1.0(6)$ & $\mathrm{C} 8-\mathrm{C} 11-\mathrm{N} 2-\mathrm{C} 12$ & $-178.8(4)$ \\
\hline $\mathrm{N} 3-\mathrm{C} 15-\mathrm{C} 16-\mathrm{C} 17$ & $-178.5(4)$ & $\mathrm{C} 17-\mathrm{C} 12-\mathrm{N} 2-\mathrm{C} 11$ & $173.3(4)$ \\
\hline $\mathrm{C} 14-\mathrm{C} 15-\mathrm{C} 16-\mathrm{C} 17$ & $1.8(6)$ & $\mathrm{C} 13-\mathrm{C} 12-\mathrm{N} 2-\mathrm{C} 11$ & $-8.6(6)$ \\
\hline $\mathrm{C} 13-\mathrm{C} 12-\mathrm{C} 17-\mathrm{C} 16$ & $0.7(6)$ & $\mathrm{C} 14-\mathrm{C} 15-\mathrm{N} 3-\mathrm{C} 18$ & $3.3(7)$ \\
\hline $\mathrm{N} 2-\mathrm{C} 12-\mathrm{C} 17-\mathrm{C} 16$ & $178.9(4)$ & $\mathrm{C} 16-\mathrm{C} 15-\mathrm{N} 3-\mathrm{C} 18$ & $-176.3(4)$ \\
\hline $\mathrm{C} 15-\mathrm{C} 16-\mathrm{C} 17-\mathrm{C} 12$ & $-1.7(7)$ & $\mathrm{C} 14-\mathrm{C} 15-\mathrm{N} 3-\mathrm{C} 20$ & $-178.2(4)$ \\
\hline $\mathrm{C} 10-\mathrm{C} 5-\mathrm{N} 1-\mathrm{C} 2 \mathrm{~A}$ & $34.3(15)$ & $\mathrm{C} 16-\mathrm{C} 15-\mathrm{N} 3-\mathrm{C} 20$ & $2.1(6)$ \\
\hline $\mathrm{C} 6-\mathrm{C} 5-\mathrm{N} 1-\mathrm{C} 2 \mathrm{~A}$ & $-144.8(15)$ & $\mathrm{C} 19-\mathrm{C} 18-\mathrm{N} 3-\mathrm{C} 15$ & $-86.1(5)$ \\
\hline $\mathrm{C} 10-\mathrm{C} 5-\mathrm{N} 1-\mathrm{C} 3 \mathrm{~A}$ & $-146.5(10)$ & $\mathrm{C} 19-\mathrm{C} 18-\mathrm{N} 3-\mathrm{C} 20$ & $95.3(5)$ \\
\hline $\mathrm{C} 6-\mathrm{C} 5-\mathrm{N} 1-\mathrm{C} 3 \mathrm{~A}$ & $34.4(12)$ & $\mathrm{C} 21-\mathrm{C} 20-\mathrm{N} 3-\mathrm{C} 15$ & $-86.4(6)$ \\
\hline
\end{tabular}




\section{supporting information}

$\mathrm{C} 10-\mathrm{C} 5-\mathrm{N} 1-\mathrm{C} 2 \quad-11.9(9) \quad \mathrm{C} 21-\mathrm{C} 20-\mathrm{N} 3-\mathrm{C} 18 \quad 92.1(5)$

Hydrogen-bond geometry $\left(A,{ }^{\circ}\right)$

$\mathrm{Cg} 1$ and $\mathrm{Cg} 2$ are the centroids of the $\mathrm{C} 5-\mathrm{C} 10$ and $\mathrm{C} 12-\mathrm{C} 17$ rings, respectively.

\begin{tabular}{lllll}
\hline$D-\mathrm{H} \cdots A$ & $D-\mathrm{H}$ & $\mathrm{H} \cdots A$ & $D \cdots A$ & $D-\mathrm{H} \cdots A$ \\
\hline $\mathrm{O} 1-\mathrm{H} 1 \cdots \mathrm{N} 2$ & $0.86(2)$ & $1.81(4)$ & $2.563(5)$ & $144(6)$ \\
$\mathrm{C} 18-\mathrm{H} 18 A \cdots C g 2^{\mathrm{i}}$ & 0.97 & 2.92 & $3.660(5)$ & 134 \\
$\mathrm{C} 1 A-\mathrm{H} 1 A 1 \cdots C g 1^{\mathrm{ii}}$ & 0.96 & 2.80 & $3.49(4)$ & 130 \\
\hline
\end{tabular}

Symmetry codes: (i) $-x+5 / 2,-y-1, z+1 / 2$; (ii) $-x-1, y+3 / 2,-z+1 / 2$. 